\title{
Theory of Low-Temperature Hall Effect in Electron-Doped Cuprates
}

\author{
Jie Lin and A. J. Millis \\ Department of Physics, Columbia University, 538 West 120th Street, New York, NY 10027
}

\begin{abstract}
A mean field calculation of the $T \rightarrow 0$ limit of the Hall conductance of electron-doped cuprates such as $\mathrm{Pr}_{2-x} \mathrm{Ce}_{x} \mathrm{CuO} \mathrm{O}_{4+\delta}$ is presented. The data are found to be qualitatively consistent with the reconstruction of the Fermi surface expected upon density wave ordering. The magnitude of the density wave gap is found to be large. The Hall resistance exhibits a nonanalyticity at the quantum critical point for density wave ordering, but the amplitude of the anomaly is found to be unobservably small. The quantum critical contribution to $R_{H}(B)$ is determined. Quantitative discrepancies between calculation and data remain, suggesting that the experimental doping is not identical to the $C e$ concentration $x$.
\end{abstract}

PACS numbers: 74.72.Jt, 74.25.Fy

\section{INTRODUCTION}

The unusual properties of high temperature cuprate superconductors continue to challenge our understanding of the physics of electrons in metals. The related ideas of "quantum criticality" $\underline{1}$ and density wave (or "stripe") order and fluctuations 2.3 have been much debated, but in the hole-doped cuprates the discussion has been inconclusive. Further, while it has been proposed ${ }^{1.4}$ that many of the anomalous properties of the materials could be explained by a quantum critical point at or near "optimal doping", there is no unambiguous evidence of long ranged order setting in near optimal doping in the holedoped cuprates.

The electron-doped cuprates offer a new perspective on these issues. In these materials commensurate $(\pi, \pi)$ spin density wave order has been detected by muon spin rotation ${ }^{5}$ and neutron scattering measurements $\frac{6.7}{6}$ The order exists over a wide range of dopings and vanishes at a critical doping $x_{c}$ near the "optimal" doping $x=0.16$. Thus, in this material an unambiguous quantum critical point exists, separating an apparently disordered phase from a phase with a well-defined $((\pi, \pi)$ commensurate magnetic) order. It is therefore of interest to examine the effect of the order and criticality on material properties. In this paper we consider the low temperature Hall resistance, which is sensitive to the rearrangment of the Fermi surface caused by the onset of the spin density wave order. We apply to the electron-doped cuprate case a meanfield analysis presented previously in the literature,, 8.10 and compare the results to experiments 11.12

The rest of this paper is organized as follows. Sec. [II defines the model to be studied. The formal solution to Boltzmann equation with relaxation time approximation for 2-dimensional systems is derived in Sec. III Sec. IV gives the numerical results for $R_{H}$ in weak magnetic field and compares them to experimental data. In Sec. $\nabla$ we analyze our formal solution in the critical region, $x \lesssim$ $x_{c}$. The sensitivity of our numerical results to variation of parameters is studied in Sec. VI The possibility of explaining the $x>x_{c}$ experimental results by calculating the real part of the self energy due to spin fluctuations is investigated in Sec. VII We conclude our calculations and discuss our results in Sec. VIII]

\section{MODEL}

We consider electrons moving in a square lattice of unit lattice constant with dispersion

$$
\begin{aligned}
\varepsilon_{p}=-2 t_{1}\left(\cos p_{x}+\cos p_{y}\right) & +4 t_{2} \cos p_{x} \cos p_{y} \\
& -2 t_{3}\left(\cos 2 p_{x}+\cos 2 p_{y}\right)
\end{aligned}
$$

The "canonical" values of the band parameters are $t_{1}=$ $0.38 \mathrm{eV}, t_{2}=0.32 t_{1}$ and $t_{3}=0.5 t_{2}$. As will be shown below, our main results are not sensitive to the precise parameter choices. We are interested in electron dopings, corresponding to two dimensional carrier densities per unit cell $n=1+x>1$.

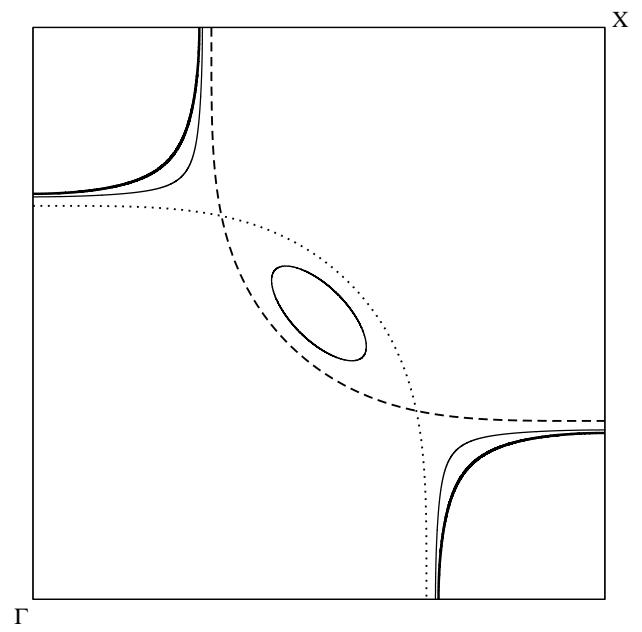

FIG. 1: Fermi surfaces calculated from $E q$ [ for $x=0.15$, band parameters $t_{1}=0.38 \mathrm{eV}, t_{2}=0.32 t_{1}, t_{3}=0.5 t_{2}$ and backscattering ("gap") values $\Delta=0$ (dashed line), $\Delta=0.2 \mathrm{eV}$ (light solid line) and $\Delta=0.4 \mathrm{eV}$ (heavy solid line). The dotted line is given by $\varepsilon_{p+Q}=\mu(\Delta=0)$.

We assume that for $x$ less than a critical value $x_{c}$ a commensurate spin density wave (SDW) order occurs, so 
the electrons are subject to a coherent backscattering of amplitude $\Delta$ and wavevector $\vec{Q}=(\pi, \pi)$ which doubles the unit cell, changes the dispersion to

$$
E_{p}^{ \pm}=\frac{1}{2}\left(\varepsilon_{p}+\varepsilon_{p+Q} \pm \sqrt{\left(\varepsilon_{p}-\varepsilon_{p+Q}\right)^{2}+4 \Delta^{2}}\right)
$$

and reconstructs the Fermi surface. If $\Delta=0$ then (at the relevant dopings $x$ ) the Fermi surface consists of one large hole surface centered at the $X$ point $(\pi \cdot \pi)$ (dashed line in Fig (1). If $\Delta \neq 0$ then the Fermi surface is reconstructed. The details depend on the band filling and the magnitude of $\Delta$. If the chemical potential $\mu$ is such that $\Delta<\Delta^{*}(\mu)=\varepsilon_{(\pi / 2, \pi / 2)}-\mu=4 t_{3}-\mu$ then both bands cross the Fermi level and the Fermi surface involves two symmetry-inequivalent hole pockets centered at $(\pi / 2, \pm \pi / 2)$ and one electron pocket centered at $(0, \pi)$. One of the hole pockets and two portions of the electron pocket are shown as the light solid lines in Fig 1 However, if $\Delta>\Delta^{*}(\mu)$, the lower band is completely filled and only the electron pocket remains (heavy solid line in Fig 1). For the parameters used in Fig 1 $\Delta^{*} \approx 0.26 \mathrm{eV}$.

As $x$ is decreased below $x_{c}$ we assume that the gap magnitude increases. However, the band filling and hence the chemical potential decreases. Therefore, depending on the relative growth (with decreasing $x$ ) of $\mu$ and $\Delta$ either the hole pockets vanish at a critical $x=x_{1}$, or it persists to $x=0$. The comparison to data given below suggest that the former circumstance obtains in the actual materials, and that indeed the gap opens so rapidly that $x_{1}$ is of order 0.12 , not much less than $x_{c} \approx 0.16$.

\section{CALCULATION OF HALL RESISTANCE: FORMALISM}

We require the $T \rightarrow 0$ transport coefficients. In the $T \rightarrow 0$ limit the only scattering is due to impurities, and we assume a Boltzmann equation description is adequate, so that the physics is described by a momentum space distribution function $g(p)=f(p)+h_{p}$ with $f(p)$ the Fermi-Dirac distribution. We also adopt the relaxation time approximation, linearize in the applied electric field $\vec{E}$ and take the $T \rightarrow 0$ limit so that for each band $b$, the Boltzmann equation becomes $(e=|e|)$ :

$$
-e \vec{E} \cdot \vec{v}^{b} \delta\left(E_{p}^{b}-\mu\right)=\left(\frac{\hbar}{\tau_{p}}+\frac{e}{c} \vec{B} \cdot\left(\vec{v}_{p}^{b} \times \frac{\partial}{\partial \vec{p}}\right)\right) h_{p}^{b}
$$

We are interested in two dimensional electrons. We take $B \| \hat{z}$ and note that $\vec{B} \cdot\left(\vec{v}_{p}^{b} \times \frac{\partial}{\partial \vec{p}}\right)$ corresponds to a derivative along the Fermi contour. Defining $s$ to be the coordinate along the Fermi arc, noting that the structure of the equation implies

$$
h_{p}^{b}=\delta\left(E_{p}^{b}-\mu\right) h^{b}(s)
$$

and temporarily dropping, for ease of writing, the band superscript b allow us to rewrite Eq 3 as

$$
\left[1+a(s) \partial_{s}\right] h(s)=E(s)
$$

with $\left(l=v \tau\right.$ is the mean free path, and $\Phi_{0}=h c / 2 e$ is the superconducting flux quantum):

$$
\begin{aligned}
& a(s)=\frac{e \tau(s) B|v(s)|}{\hbar c}=\frac{\pi l(s) B}{\Phi_{0}} \\
& E(s)=-\frac{e \tau(s) \vec{E} \cdot \vec{v}}{\hbar}
\end{aligned}
$$

Eq 5 may be formally solved ${ }^{13}$ in terms of the Green function $K\left(s, s^{\prime}\right)$ satisfying

$$
\left[1 / a(s)+\partial_{s}\right] K\left(s, s^{\prime}\right)=\delta\left(s-s^{\prime}\right)
$$

with boundary condition

$$
K\left(s \rightarrow s^{\prime+}, s^{\prime}\right)-K\left(s \rightarrow s^{\prime-}, s^{\prime}\right)=1
$$

as

$$
h(s)=\oint d s^{\prime} K\left(s, s^{\prime}\right) E\left(s^{\prime}\right) / a\left(s^{\prime}\right)
$$

The currents longitudinal and transverse to $\vec{E}$ may then be constructed by averaging over the velocity as usual. Assuming a square lattice, restoring the factors of velocity and the band label (if necessary), and symmetrizing or antisymmetrizing for $\sigma_{x x, x y}$, we find

$$
\begin{aligned}
& \sigma_{x x}=\sigma_{Q} \oint \frac{d s d s^{\prime}}{p_{B}^{2}} K\left(s, s^{\prime}\right) \cos \left[\phi(s)-\phi\left(s^{\prime}\right)\right] \\
& \sigma_{x y}=\sigma_{Q} \oint \frac{d s d s^{\prime}}{p_{B}^{2}} K\left(s, s^{\prime}\right) \sin \left[\phi(s)-\phi\left(s^{\prime}\right)\right]
\end{aligned}
$$

where $\sigma_{Q} \equiv e^{2} / \hbar$ is conductance quantum, $p_{B} \equiv 2 \pi / l_{B}$ is proportional to the inverse of the magnetic length $l_{B} \equiv$ $\left(\Phi_{0} / \pi B\right)^{1 / 2}$, and $\phi$ is the polar angle of Fermi velocity on the corresponding Fermi contours. The formulae are valid as long as magnetic breakdown can be neglected. A criterion for this is given below.

The problem has several momentum scales. Obtaining a non-vanishing Hall coefficient requires breaking of particle-hole symmetry, either by a variation of scattering rate, a Fermi surface curvature, or a variation of velocity. All of these phenomena are characterized by momentum scales of the order of the size of the Brillouin zone; we refer to these scales generically as $p_{0}$. We take these to be large, so we may neglect Landau quantization. A second important scale $p_{\Delta}=\Delta / v_{F}$ is set by the SDW backscattering amplitude, $\Delta$, and vanishes as $\Delta \rightarrow 0$. A third scale is the range, $a$, of the kernel, $K$ (Eq [6]). We distinguish weak field $\left(a<p_{\Delta}\right)$ and strong field $\left(a>p_{\Delta}\right)$ regimes. A fourth significant scale is the inverse of the mean free path, $l^{-1}$. The results we present are valid for $p_{0} \gg p_{\Delta}, a, l^{-1}$. In addition, we expect disorder to modify the SDW behavior significantly for $p_{\Delta} l \sim 1$; so our results do not apply for $\Delta<v_{F} / l$. In this case we may neglect the periodicity and write

$$
K\left(s, s^{\prime}\right)=\exp \left[-\int_{s^{\prime}}^{s} \frac{d x}{a(x)}\right] \Theta\left(s-s^{\prime}\right)
$$




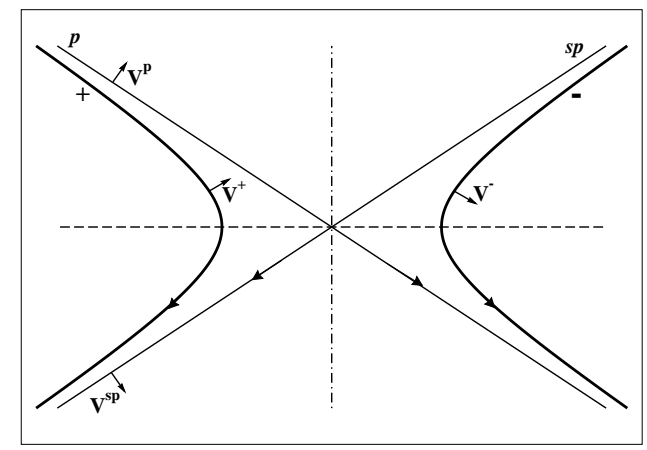

FIG. 2: Local view of the Fermi surface in the vicinity of one of crossing points, $\varepsilon_{p}=\varepsilon_{p+Q}=\mu$, shown in Fig 1 . $p$, sp are respectively the Fermi lines for the normal state and the normal state Fermi line shifted by $\vec{Q}$. The curvature has been neglected. + denotes the electron pocket of SDW state anddenotes the hole pocket. Arrows along Fermi lines show the circulation direction determined by $\mathbf{B} \times \mathbf{v}$ with $\mathbf{B} \|-\mathbf{z}$. They also indicate the direction of increase in arclength coordinate, s. $V^{p}, V^{s p}, V^{+}$and $V^{-}$are Fermi velocities along corresponding Fermi lines. The dashed line is the diagonal of the first quadrant of Brillouin zone connecting $(0, \pi)$ and $(\pi, 0)$. The dot-dashed line is perpendicular to the dashed line: if the normal state energy dispersion is linearized, and the momentum dependence of velocity is neglected, the problem is symmetric under reflection through the dot-dashed line.

Finally, we note that all of the formulae are derived on the assumption that a state evolves along a given Fermi contour and does not jump to another one, i.e., that magnetic breakdown may be neglected. By applying the considerations of Ref. 14 to the model studied here, we find the condition for absence of magnetic breakdown is, in order of magnitude, that the inverse of the magnetic length $p_{B}=\left(\pi B / \Phi_{0}\right)^{-1 / 2} 2 \pi$ be less than the gap momentum scale $p_{\Delta}$. Our results are only valid if $a, p_{\Delta}>p_{B}$. The condition that breakdown occurs at a field higher than the weak-strong field crossover is $p_{\Delta} l>1$, which as noted above is a necessary condition for the validity of the approach.

\section{WEAK FIELD LIMIT: EVALUATION AND COMPARISON TO DATA}

In this section we analyse in detail the case in which the field is weak enough that $a$ is small relative to all scales in the problem. In this case the $s^{\prime}$ integrals in Eqs 1112] are dominated by $s^{\prime} \approx s$ and may be performed, leaving

$$
\begin{aligned}
\sigma_{x x} & =\sigma_{Q} \frac{1}{4 \pi^{2}} \oint d s l(s) \\
\sigma_{x y} & =\sigma_{Q} \frac{1}{4 \pi^{2}} \oint d s\left(l(s) / l_{B}\right)^{2} \frac{\partial \phi(s)}{\partial s}
\end{aligned}
$$

for the longitudinal and Hall conductance per plane. Eq 14] is exactly the Drude result (average of mean free path over the Fermi arc), while Eq [15] reproduces Ong's result 15

$$
\sigma_{x y}=\sigma_{Q} \frac{B}{\Phi_{0}} \frac{1}{2 \pi} \oint d \vec{l} \times \vec{l} \cdot \hat{\mathbf{z}} / 2
$$

Further analysis requires information about the scattering rates. The two commonly made assumptions are:

- mean free path is constant over the Fermi surface

- scattering rate is constant over Fermi surface

The constant scattering rate assumption may be derived theoretically by assuming weak (Born approximation) point-like scatterers. Constant mean free path is more appropriate for very strong (unitary) scattering.

Ong 15 shows that the constant mean free path approximation implies that the Hall conductance for each Fermi surface pocket is a constant, depending only on the value of the mean free path and the sign (electron- or hole-like) of the Fermi pocket. Therefore, in the constant mean free path approximation, $\sigma_{x y}$ does not change as $x$ is decreased through $x_{c}$. The reconstruction of the Fermi surface means that one goes from one hole pocket $\left(\sigma_{x y} \propto l^{2}\right)$ to two hole pockets $\left(\sigma_{x y} \propto 2 l^{2}\right)$ and one electron pocket $\left(\sigma_{x y} \propto-l^{2}\right)$ so that the total $\sigma_{x y} \propto l^{2}(2-1)=l^{2}$. However, at $x=x_{1}$ the hole pockets vanish, and in the constant mean free path approximation the Hall coefficient would jump discontinuously from a value close to the $R_{H} \sim 1 /(1-x)$ implied by the initial, large, holelike surface, to the value $R_{H} \sim-1 / x$ implied by a single electron-like pocket. This behavior is in evident disagreement with data, 11.12 which indicates instead a smooth change beginning at $x \approx 0.16$, which is approximately the doping at which antiferromagnetism sets in $\underline{7}^{7}$

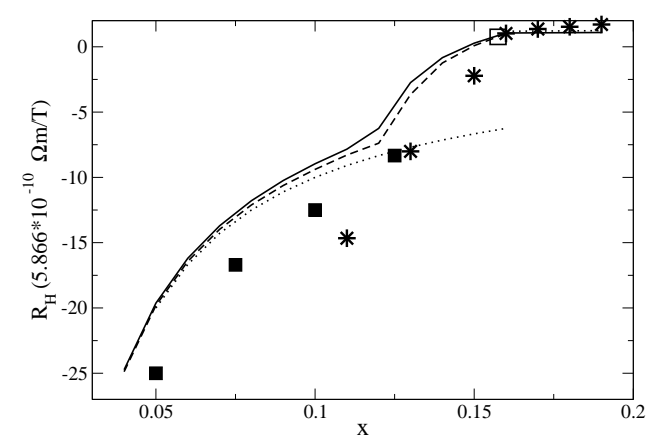

FIG. 3: Solid line: Hall coefficient calculated from Eqs 14 16] for parameters $t_{1}=0.38 \mathrm{eV}, t_{2}=0.32 t_{1}, t_{2}=0.5 t_{2}$ and $\Delta(x)[e V]=0.6 \sqrt{1-x / 0.16}$. Long dashed line: Hall coefficient calculated for parameters $t_{1}=0.38 \mathrm{eV}, t_{2}=0.32 t_{1}$, $t_{2}=0.075 \mathrm{eV}$ and $\Delta(x)[\mathrm{eV}]=0.7 \sqrt{1-x / 0.16}$. They are calculated with constant $1 / \tau$ approximation. Dotted line: curve $R_{H}=-1 / x$ for $x<0.16$ and $R_{H}=1 /(1-x)$ for $x>0.16$. Stars: experimental data ${ }^{11}$ Solid squares: experimental data. ${ }^{12}$ The open box shows the approximate size of the region for which the weak field-strong field crossover may be observable with present accessible fields. 
We therefore turn to the constant $1 / \tau$ approximation. In this case, $\sigma_{x y}$ for each pocket is weighted by the square of the Fermi velocity for that pocket and more variation occurs. The solid line and long dashed line in Fig 3) show typical results obtained by evaluating Eqs 1416 in the constant scattering rate approximation and assuming a backscattering amplitude growing proportional to $\sqrt{x_{c}-x}$. One sees that for $x>x_{c}$ the Hall resistance is hole-like and depends only very weakly on $x$. At $x=x_{c}$ a nonanalyticity occurs, and $R_{H}$ begins to decrease. For the parameters chosen, $x_{1} \sim 0.12$. At this doping the hole pockets vanish, a further change in curvature occurs, and $R_{H}$ approximates very closely the $1 / x$ behavior expected from the single remaining electron pocket (shown as the dotted line).

Fig 3 also shows, as stars and heavy squares, the experimental results ${ }^{11.12}$ These data are in qualitative agreement with calculation, but important quantitative differences occur. At the lowest doping (nominal carrier concentration $x=0.11$ for stars 11 and $x=0.05$ for heavy squares 12 ) the measured $R_{H}$ is substantially below both the calculated value and the $1 / x$ curve. It seems to us essentially impossible to obtain a Hall resistance satisfying $\left|R_{H}\right|>|1 / x|$; we therefore suggest that the nominal doping level, $x$, differs from the actual doping. The presently available data do not allow us to locate the point $x_{1}$ with precision, but strongly suggest that $x_{1} \geq 0.1$.

\section{CRITICAL BEHAVIOR}

In this section, we study in more detail the behavior of Hall conductance $\sigma_{x y}$ in the vicinity of $x=x_{c}$. When the system is tuned through the critical point (which we believe to be $x_{c} \approx 0.16$ in the electron-doped cuprates), the coherent backscattering introduces reconstruction of Fermi surface, changes its local curvature, and thus affects the Hall conductance. The weak field limit was previously studied by Bazaliy et a $a^{116}$ in the context of the 3d SDW compound $\mathrm{Cr}$. They obtained a result equivalent to our Eq 31] and a qualitative result equivalent to the weak field limit of our Eq 22. And the strong field limit was studied by Fenton and Schofield, $\frac{17}{1}$ who found a result equivalent to our Eqs 3233 . The two dimensional nature of the present problem allows us to carry the analysis further, obtaining prefactors for $\sigma_{x y}$ in the weak field limit and a treatment of the weak to strong field crossover.

To study the vicinity of the critical point, we use Eq 12 to write an equation for the change in the Hall conductance as the gap is opened, $\delta \sigma_{x y}=\sigma_{x y}(\Delta)-\sigma_{x y}(\Delta=$ $0)$. For small $\Delta$ the important changes in the Fermi surface occur near the points where the $\Delta=0$ Fermi surface crosses its translation by $\mathbf{Q}$, i.e. where $\varepsilon_{p}=\varepsilon_{p+Q}=\mu$. One of these crossing points is shown in Fig 2] The change of chemical potential is second order effect of $\Delta$, and thus neglected in our analysis. To simplify notation, in this section, we measure $\mu$ from its value at critical- ity. Thus the SDW Fermi surface with backscattering potential $\Delta, E_{p}^{ \pm}=0$, can be written as $\varepsilon_{p} \varepsilon_{p+Q}=\Delta^{2}$. Any change in the scattering rate is similarly of order $\Delta^{2}$ and is neglected. Near such a crossing point we may write $\delta \sigma_{x y}$ in terms of the contributions from the two SDW bands $E_{p}^{ \pm}$and the original Fermi surface, $p$, and its image under translation by $\mathbf{Q}, s p$, as

$$
\begin{array}{r}
\delta \sigma_{x y} \sim \int_{-\infty}^{\infty} d s d s^{\prime}\left(\sum_{b= \pm} K_{b}\left(s, s^{\prime}\right) \sin \left[\phi_{b}(s)-\phi_{b}\left(s^{\prime}\right)\right]\right. \\
\left.-\sum_{a=p, s p} K_{a}\left(s, s^{\prime}\right) \sin \left[\phi_{a}(s)-\phi_{a}\left(s^{\prime}\right)\right]\right)
\end{array}
$$

We shall see that this integral is dominated by $s, s^{\prime}$ close to the crossing point, with "close" determined by the larger of $a$ (evaluated at the crossing point) and $p_{\Delta}$, so that we have neglected the fact that the Fermi surface closes on itself and have extended the integration to infinity.

We analyse Eq [17 by expanding the energy dispersions about the crossing point. In the first approximation, one may consider taking $\tau$ to be constant and linearizing the dispersions about the crossing point, writing $\varepsilon_{p}=\mathbf{v}^{p} \cdot \delta \mathbf{p}$ and $\varepsilon_{p+Q}=\mathbf{v}^{s p} \cdot \delta \mathbf{p}$, with $\mathbf{v}^{p, s p}$ constant. In this approximation, the $\Delta=0$ Fermi surface and its image are straight lines, so $\phi^{p, s p}$ are independent of $s$, so the $\Delta=0$ contribution vanishes, and the two SDW bands are mirror images of each other with respect to the dot-dashed line in Fig [2] so $K_{+}(s)=K_{-}(s)$ and $\phi_{+}(s)-\phi_{+}\left(s^{\prime}\right)=-\left(\phi_{-}(s)-\phi_{-}\left(s^{\prime}\right)\right)$, so that $\delta \sigma_{x y}$ vanishes. Obtaining a non-zero $\delta \sigma_{x y}$ requires consideration of effects which break this mirror symmetry. These include variation of $\tau$ or $\left|v^{p}\right|$ with momentum as well as curvature of the $\Delta=0$ Fermi surface. Formally, all of these effects are of order $p_{0}^{-1}$, and we work to leading order in this quantity. Then we may write

$$
a^{ \pm}(s)=a(s)[1 \pm \delta a(s)]
$$

where $a(s)$ is the corresponding function in presence of mirror symmetry, and $\delta a(s)$ represents the broken mirror symmetry, and thus is of order $\mathcal{O}\left(1 / p_{0}\right)$. In terms of these functions:

$$
\begin{aligned}
K_{ \pm}\left(s, s^{\prime}\right) & \approx K\left(s, s^{\prime}\right)\left(1 \pm \int_{s^{\prime}}^{s} d x \frac{\delta a(x)}{a(x)}\right) \\
& =K\left(s, s^{\prime}\right)(1 \pm \delta K)
\end{aligned}
$$

We also have:

$$
\phi_{+}(s)-\phi_{+}\left(s^{\prime}\right)+\phi_{-}(s)-\phi_{-}\left(s^{\prime}\right)=f\left(s, s^{\prime}\right)
$$

where $f$ is also of order $1 / p_{0}$. Thus, Eq 17 can be expanded as:

$$
\begin{aligned}
\delta \sigma_{x y} \sim \int d s d s^{\prime}\{ & 2 K\left(s, s^{\prime}\right) f\left(s, s^{\prime}\right) \cos \left[\phi_{+}(s)-\phi_{+}\left(s^{\prime}\right)\right] \\
+ & 2 K\left(s, s^{\prime}\right) \delta K\left(s, s^{\prime}\right) \sin \left[\phi_{+}(s)-\phi_{+}\left(s^{\prime}\right)\right] \\
& \left.-K_{p}\left(s, s^{\prime}\right) g\left(s, s^{\prime}\right)-K_{s p}\left(s, s^{\prime}\right) g\left(s, s^{\prime}\right)\right\}
\end{aligned}
$$


with $g\left(s, s^{\prime}\right)$ of order $1 / p_{0}$. In obtaining this equation, we have noticed that $f\left(s, s^{\prime}\right), \delta K$ and $g\left(s, s^{\prime}\right)$ are already of order $1 / p_{0}$, so that all the remaining quantities can be taken from their unperturbed counterparts. We also notice that $a^{p}(s)=a^{s p}(s)$, and in this approximation they are constant, denoted by $a^{p}$ in the following discussion.

The detailed analysis of this equation is somewhat invovled, except for the strong magnetic field limit to be discussed below, partly because it is not easy to obtain an analytic relationship between the arclength coordinates $s$ and momentum coordinate-p for SDW Fermi line. We shall argue that this $\delta \sigma_{x y}$ has a scaling form:

$$
\delta \sigma_{x y}=C(B \Delta) F\left(a^{p} / p_{\Delta}\right)
$$

such that $F(0)=1$ and $F(x \rightarrow \infty) \propto x$, with the prefactor $C$ of order $1 / p_{0}$, which will be computed in the special case of constant relaxation time in the following, and the exact form of $F$ depending on the manner in which the symmetry between + and - branches is broken.

It has been noted by Bazaliy et $a^{116}$ that in the vicinity of the crossing points, $\mathbf{p}$ can be parametrized by $\varepsilon_{p}$ and $\varepsilon_{p+Q}$ : first expanding the dispersions

$$
\begin{aligned}
\varepsilon_{p} & =\mathbf{v}^{p \star} \cdot \delta \mathbf{p}+\frac{1}{2} m_{i j} \delta p_{i} \delta p_{j}+O\left(\delta p^{3}\right) \\
\varepsilon_{p+Q} & =\mathbf{v}^{s p \star} \cdot \delta \mathbf{p}+\frac{1}{2} n_{i j} \delta p_{i} \delta p_{j}+O\left(\delta p^{3}\right)
\end{aligned}
$$

where, $\delta \mathbf{p}=\mathbf{p}-\mathbf{p}^{\star}, \mathbf{v}^{p, s p \star}=\mathbf{v}^{p, s p}\left(\mathbf{p}^{\star}\right) \equiv\left(\nabla_{p} \varepsilon_{p, p+Q}\right)^{\star}$, $m_{i j}=\left(\partial^{2} \varepsilon_{p} / \partial p_{i} \partial p_{j}\right)^{\star}, n_{i j}=\left(\partial^{2} \varepsilon_{p+Q} / \partial p_{i} \partial p_{j}\right)^{\star}$, and the superscript $\star$ stands for the corresponding value evaluated at the crossing point, and then inverting them

$$
\delta \mathbf{p}=\mathbf{u}_{1} \varepsilon_{p}+\mathbf{u}_{2} \varepsilon_{p+Q}=\left(\mathbf{u}_{1}+\frac{\Delta^{2}}{\varepsilon_{p}^{2}} \mathbf{u}_{2}\right) \varepsilon_{p}
$$

with $\mathbf{u}_{1}=\frac{\mathbf{v}^{s p \star} \times\left[\mathbf{v}^{p \star} \times \mathbf{v}^{s p \star}\right]}{\left(\mathbf{v}^{p \star} \times \mathbf{v}^{s p}\right)^{2}}$ and $\mathbf{u}_{2}=\frac{\mathbf{v}^{p \star} \times\left[\mathbf{v}^{s p \star} \times \mathbf{v}^{p \star}\right]}{\left(\mathbf{v}^{p \star} \times \mathbf{v}^{s p \star}\right)^{2}}$. From Eq 24]:

$$
d \mathbf{p}=\mathbf{u}_{1} d \varepsilon_{p}+\mathbf{u}_{2} d \varepsilon_{p+Q}=\left(\mathbf{u}_{1}-\frac{\Delta^{2}}{\epsilon_{p}^{2}} \mathbf{u}_{2}\right) d \epsilon_{p}
$$

where the second equality of Eqs 2425] only holds on the SDW Fermi surface. Thus,

$$
\begin{aligned}
\left|\frac{d s}{d \varepsilon_{p}}\right|=\frac{1}{\left|\mathbf{v}^{p} \times \mathbf{v}^{s p}\right|}\left\{\left(v^{s p}\right)^{2}\right. & +\left(v^{p}\right)^{2}\left(\frac{\Delta^{2}}{\varepsilon_{p}^{2}}\right)^{2} \\
+ & \left.2\left(\frac{\Delta^{2}}{\varepsilon_{p}^{2}}\right) \mathbf{v}^{p} \cdot \mathbf{v}^{s p}\right\}^{1 / 2}
\end{aligned}
$$

And the SDW Fermi velocities are:

$$
\mathbf{v}^{ \pm}(\mathbf{p})=\frac{\Delta^{2}}{\varepsilon_{p}^{2}+\Delta^{2}} \mathbf{v}^{p}(\mathbf{p})+\frac{\varepsilon_{p}^{2}}{\varepsilon_{p}^{2}+\Delta^{2}} \mathbf{v}^{s p}(\mathbf{p})
$$

Now return to Eq 21. $a(s)=\pi B \tau_{0} v(s) / \Phi_{0}$, where $\tau_{0}$ is the relaxation time at crossing point, $v(s)$ is the SDW
Fermi velocity when mirror symmetry is present, in which case $\mathbf{v}^{p}$ and $\mathbf{v}^{s p}$ in Eqs [26 27] are constant vectors. Substituting this and Eq [26] into Eq 21], scaling energy variables in unit of $\Delta$, and defining $\mathcal{B}=B\left(v_{F}^{2} \pi \tau_{0} \sin \Delta \phi / \Phi_{0}\right)$, where $v_{F}=v^{p, s p}$ and $\Delta \phi$ is the angle made by the normal state Fermi velocities, $\mathbf{v}^{p}$ and $\mathbf{v}^{s p}$, shown in Fig 2 we can obtain the above scaling form Eq 22. In the following, we shall discuss Eq 22 in two limits: weak magnetic field, $a^{p} \ll p_{\Delta}$, and strong magnetic field, $a^{p} \gg p_{\Delta}$.

In weak magnetic field, we can start from Eq [16. Both $\mathbf{v}^{p}$ and $\tau$ can vary in momentum space. To lowest order, these effects are additive. If we keep the $\mathbf{p}$-dependence of relaxation time $\tau$, assuming its dependence has form:

$$
\tau_{p}=\tau_{0}\left[1+\mathcal{A}\left(\varepsilon_{p}+\varepsilon_{p+Q}\right)\right]
$$

and keep velocity of normal state, $\mathbf{v}^{p}$ and $\mathbf{v}^{s p}$, to be constant, Eq [16] can be evaluated for each Fermi pocket, giving

$$
\delta \sigma_{x y}=\sigma_{Q} 4 \tau_{0}^{2} \mathcal{A} \frac{B \Delta}{\Phi_{0}} \hat{\mathbf{z}} \cdot\left[\mathbf{v}^{p} \times \mathbf{v}^{s p}\right]
$$

In the case in which the normal state velocities $\mathbf{v}^{p}$ and $\mathbf{v}^{s p}$ are $\mathbf{p}$-dependent and relaxation time $\tau$ is constant, differentiating Eq 23], substituting Eq 24] into it, and defining vectors:

$$
\begin{aligned}
\vec{\eta}_{1}^{p} & =\left(m_{11} u_{1 x}+m_{12} u_{1 y}\right) \hat{\mathbf{x}}+\left(m_{21} u_{1 x}+m_{22} u_{1 y}\right) \hat{\mathbf{y}} \\
\vec{\eta}_{2}^{p} & =\left(m_{11} u_{2 x}+m_{12} u_{2 y}\right) \hat{\mathbf{x}}+\left(m_{21} u_{2 x}+m_{22} u_{2 y}\right) \hat{\mathbf{y}} \\
\vec{\eta}_{1}^{s p} & =\left(n_{11} u_{1 x}+n_{12} u_{1 y}\right) \hat{\mathbf{x}}+\left(n_{21} u_{1 x}+n_{22} u_{1 y}\right) \hat{\mathbf{y}} \\
\vec{\eta}_{2}^{s p} & =\left(n_{11} u_{2 x}+n_{12} u_{2 y}\right) \hat{\mathbf{x}}+\left(n_{21} u_{2 x}+n_{22} u_{2 y}\right) \hat{\mathbf{y}}
\end{aligned}
$$

we then have $\mathbf{v}^{b}=\mathbf{v}^{b \star}+\delta \mathbf{v}^{b}=\mathbf{v}^{b \star}+\left(\vec{\eta}_{1}^{b}+\vec{\eta}_{2}^{b}\left(\Delta / \varepsilon_{p}\right)^{2}\right) \varepsilon_{p}$ and $d \mathbf{v}^{b}=\left(\vec{\eta}_{1}^{b}-\vec{\eta}_{2}^{b}\left(\Delta / \varepsilon_{p}\right)^{2}\right) d \varepsilon_{p},(b=p, s p)$. Eq [16 then gives:

$\delta \sigma_{x y}=\sigma_{Q} \tau^{2} \frac{B \Delta}{\Phi_{0}} \hat{\mathbf{z}} \cdot\left[\left(\vec{\eta}_{1}^{p}+\vec{\eta}_{2}^{s p}+3 \vec{\eta}_{2}^{p}+3 \vec{\eta}_{1}^{s p}\right) \times\left(\mathbf{v}^{s p \star}-\mathbf{v}^{p \star}\right)\right]$

These weak magnetic field results, Eqs 29130, confirm our scaling form for $\delta \sigma_{x y}, \mathrm{Eq}$ [22, in the limit $a^{p} / p_{\Delta} \ll 1$. Also the prefactor $C$ can be calculated from these two equations. It would be of interest to study Eq [30 in more detail, since all the parameters in $\delta \sigma_{x y} / \sigma_{x y}\left(x=x_{c}+\right)$ are set by normal state energy function Eq 11. Taking $\sigma_{x y}\left(x=x_{c}+\right)$ from numerical calculation, $\delta \sigma_{x y} / \sigma_{x y}(x=$ $\left.x_{c}+\right)=-6.1 \Delta$, shown as solid line in the upper left panel of Fig 4 which is compared with the results of numerical evaluation of Eq [16] (solid circles in this panel). In weak magnetic field, the longitudinal conductivity $\sigma_{x x}$ has been treated in great detail for the case of the $3 \mathrm{~d}$ SDW transition in $C r, 16$ Following essentially the same procedure, we obtain a similar expression for $\delta \sigma_{x x}$ :

$$
\delta \sigma_{x x}=-\sigma_{Q} \frac{\tau}{\pi} \frac{\left(\mathbf{v}^{p \star}-\mathbf{v}^{s p \star}\right)^{2}}{\left|\mathbf{v}^{p \star} \times \mathbf{v}^{s p \star}\right|} \Delta
$$

Taking $\sigma_{x x}\left(x=x_{c}+\right)$ from numerical calculation, $\delta \sigma_{x x} / \sigma_{x x}\left(x=x_{c}+\right)=-2.1 \Delta$, shown as solid line in upper right panel of Fig 4 compared with the results taken 


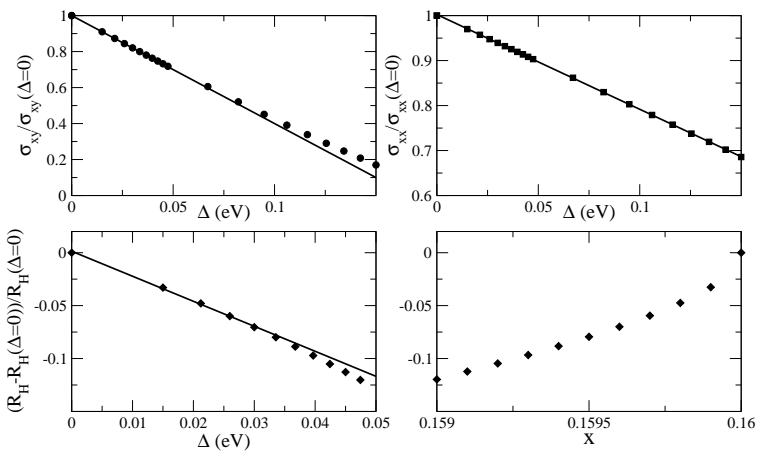

FIG. 4: Upper left: numerical results for $\sigma_{x y}(\Delta) / \sigma_{x y}(\Delta=0)$ as a function of $\Delta$ near critical doping (solid circle) and function $1.0-6.1 \Delta$ (solid line). Upper right: numerical results for $\sigma_{x x}(\Delta) / \sigma_{x x}(\Delta=0)$ as function of $\Delta$ (solid square) and function $1.0-2.1 \Delta$ (solid line). Lower left: numerical results for $R_{H}$ as a function of $\Delta$ (solid diamond) and function $-2.4 \Delta$ (solid line). Lower right: numerical results for $R_{H}$ as a function of $x$ near critical doping. The parameters are: $t_{1}=0.38 \mathrm{eV}, t_{2}=0.32 t_{1}, t_{3}=0.075 \mathrm{eV}$, and $\Delta(x)[e V]=0.6 \sqrt{1-x / 0.16}$.

from numerical evaluation of Eq 14 (solid squares). The numerical results of $R_{H} \equiv \sigma_{x y} /\left(B \sigma_{x x}^{2}\right)$ are shown as solid diamonds in the lower panels of Fig 4 The reconnection of Fermi surface suppresses both longitudinal and transverse conductivity. In the vicinity of criticality, $R_{H} / R_{H}\left(x=x_{c}+\right) \approx 1+\delta \sigma_{x y} / \sigma_{x y}\left(x=x_{c}+\right)-$ $2 \delta \sigma_{x x} / \sigma_{x x}\left(x=x_{c}+\right) \approx 1.0-1.9 \Delta$. Since $\Delta \sim \sqrt{x_{c}-x}$, we expect a square root behavior in $R_{H}(x)$. There actually is, as shown in the lower right plot of Fig 4 however, this regime is so small that it may be difficult to observe in practice.

In strong magnetic field regime $a^{p} / p_{\Delta} \gg 1$, the integration Eq 21 is dominated by $\left(s>0, s^{\prime}<0\right)$, with $f\left(s, s^{\prime}\right), g\left(s, s^{\prime}\right) \sim\left(s-s^{\prime}\right) / p_{0}, \delta a(s) \sim \operatorname{sgn} s \cdot s / p_{0}$, and $\phi_{+}(s)-\phi_{+}\left(s^{\prime}\right) \approx \Delta \phi$, then

$$
\delta \sigma_{x y}=\sigma_{Q} \frac{\left(l / l_{B}\right)^{3}}{\pi^{2} l_{B} p_{0}}[\cos \Delta \phi-1+\alpha \sin \Delta \phi]
$$

with $\alpha \sim \frac{p_{0}}{l} \frac{\partial l}{\partial s} \sim 1$ and $l$ the mean free path in normal state. This result, $\delta \sigma_{x y} \sim B^{2}$ and independent of $\Delta$, confirms our scaling function for $a^{p} / p_{\Delta} \gg 1$. To justify this result, let's consider a simple case in which the normal state velocities $\mathbf{v}^{p, s p}$ are constant, while relaxation time is assumed to be of form: Eq 28. Under this condition, the only contribution to $\delta \sigma_{x y}$ in Eq [21] comes from the second line containing $\delta K$. Changing to $\varepsilon_{p}$ variable, it gives

$$
\delta \sigma_{x y}=\sigma_{Q} \frac{\tau_{0} \mathcal{A}}{4 \pi^{2}} \mathcal{B} \Delta f(\mathcal{B} / \Delta)
$$

$f(x)$ is a function of only one variable, numerical evaluation of which gives the solid line in Fig 5 along which shown as dashed line is the linear function $f(x)=$ $2 x$, representing the calculated asymptotic behavior as

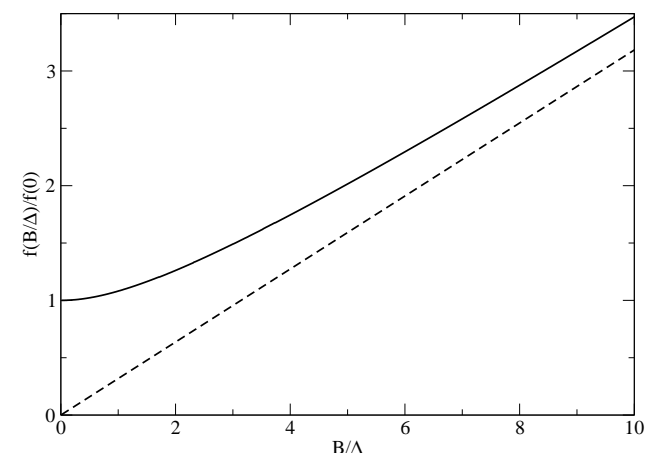

FIG. 5: The scaling function $f(B / \Delta)$ for $\delta \sigma_{x y}$ under the linearized dispersion, constant velocity approximation. The unit of $B / \Delta$ is $\mathcal{B} / \Delta$.

$x \rightarrow \infty$. The prefactor 2 is calculated by relating $\alpha$ in Eq 32 and $\mathcal{A}$ in Eq 28 in the limit $\Delta \rightarrow 0$ : $\alpha / p_{0}=\mathcal{A}\left(v_{F} \sin \Delta \phi\right) / 2$. This solid line clearly shows the expected behavior of $f(x): f(x)=1$ for $x \rightarrow 0$, and $f(x \rightarrow \infty) \propto x$, with a crossover in between. The crossover regime can be estimated by setting $\mathcal{B}=\Delta$. The relaxation time can be inferred from the $a b$ plane resistivity measurement: $\underline{\underline{11}}$ at $\mathrm{B}=10 \mathrm{~T}, \rho \sim 20 \mu \Omega \cdot \mathrm{cm}$, which gives $\tau \sim 10^{-14}$ sec. So, for $\Delta=0.1 \mathrm{eV}$, corresponding to $x_{c}-x \sim 0.005$ if we take $\Delta(x)=0.7 \sqrt{1-x / x_{c}} \mathrm{eV}$, the crossover is $B \sim \Phi_{0} \hbar \Delta /\left(v_{F}^{2} \tau\right) \sim 100 \mathrm{~T}$, where $v_{F} \approx 4 \mathrm{eV} \cdot \AA$. For these parameters, the breakdown field is slightly larger.

\section{SENSITIVITY TO PARAMETERS}

In this section we examine the sensitivity of our results to different choices of parameters.

Let us first consider the $x<x_{c}$ region. Fig [6] shows numerical results of $R_{H}$ for parameter choices: $t_{1}=0.38 \mathrm{eV}$, $t_{2}=0.32 t_{1}, t_{3}=0.075 \mathrm{eV}$ and $\Delta(x=0)=0.6 \mathrm{eV}$ (dashed line), and $t_{2}=0.32 t_{1}, t_{3}=0.5 t_{2}$ and $\Delta(x=0)=0.5 \mathrm{eV}$ (solid line), which can be compared with the solid and dashed lines in Fig [3] All of these curves share the same feature: the existence of $x_{1}$. In Fig $3 x_{1} \approx 0.12$, while in Fig 6 $x_{1} \approx 0.09$ (dashed line) and $x_{1} \approx 0.06$ (solid line). For $x>x_{1}$, both electron pocket (centered at $\left.(0, \pi)\right)$ and hole pockets (centered at $(\pi / 2, \pm \pi / 2))$ are present, and as doping decreases, both of them contribute to the decrease of $R_{H}$, while for $x<x_{1}$, the hole pockets vanish (the $E_{p}^{-}$band is filled), and thus only electron pocket contributes to $R_{H}$, which leads to the slowing down of decrease in $R_{H}$ as decreasing doping. For $x<x_{1}$, the behavior of $R_{H}$ is quite universal, approximately $\sim-1 / x$ (the Hall coefficient for free electrons, with $x$ electron density), as shown in Fig [3 Different choices of parameters change the actual value of $x_{1}$. The numerical amplitude of $R_{H}\left(x_{1}\right)$ is approximately $1 / x_{1}$, by continuity. There is still another possibility that if the opening of SDW gap is not fast enough, the hole pockets survive for 


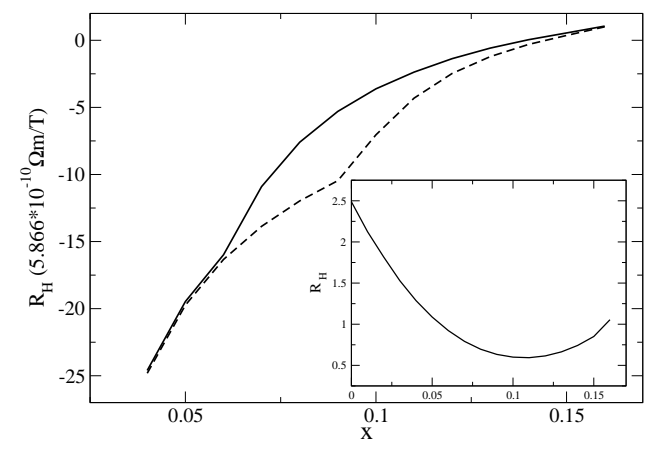

FIG. 6: Numerical results of Hall coefficient in SDW ordered state for different choices of parameters. Dashed line: $t_{1}=$ $0.38 \mathrm{eV}, t_{2}=0.32 t_{1}, t_{3}=0.075 \mathrm{eV}$ and $\Delta(x=0)=0.6 \mathrm{eV}$. Solid line: $t_{3}=0.5 t_{2}, t_{2}=0.32 t_{1}, \Delta(x=0)=0.5 \mathrm{eV}$. Inset: $R_{H}$ for $\Delta(x=0)=0.3 \mathrm{eV}$, with the same band parameters as the solid line in the main panel.

$x$ down to 0 . In this case, there is an upturn when $x$ is smaller than some value, with a much smaller relative change in the magnitude of $R_{H}$, as shown in the inset of Fig [6] We do not believe this possibility is relevant to the electron doped cuprates.

In the regime $x>x_{c}$, we can change band parameters $t_{2}$ and $t_{3}$ in Eq 11, and calculate $R_{H}$ for these choices of parameters. Fig 7 shows the results for different choices of band parameters: $t_{2}=0.32 t_{1}$ and $t_{3}=0.075 \mathrm{eV}$ (heavy solid line); $t_{2}=0.2 t_{1}$ and $t_{3}=0.5 t_{2}$ (dashed line); $t_{2}=0.45 t_{1}$ and $t_{3}=0.5 t_{2}$ (dotted line). The Fermi surfaces corresponding to these choices of parameters at $x=0.16$ are shown in Fig 8 . It can be seen that (i) different choices of band parameters do not lead to drastic difference in $R_{H}$; (ii) for each choice of band parameters, $R_{H}$ is quite insensitive to change in doping, in contrast to the experimental data, shown as solid circles; (iii) our numerical $R_{H}$ are close to $1 /(1-x)$ (the Hall coefficient for circular Fermi surface, with $1-x$ hole density), shown as light solid line in Fig 7 Both our numerical results and $1 /(1-x)$ are not close to the experimental data of Ref. 11. The difference is largest at $x=0.19$, the point farthest from criticality, where one would expect the difference to be smallest.

\section{VII. $x>x_{c}$}

Fig 7 shows that the measured $R_{H}$ at $x>x_{c}$ shows a pronounced doping dependence which is incompatible with the mean field calculation at fixed band parameters. Fig [7 also shows that a variation of the correct order of magnitude can be obtained if a doping dependence of the band parameters is assumed. In this section we investigate whether this doping dependence can be viewed as a precursor of the spin density wave instability by calculating the effect of spin fluctuations on the Fermi surface. This is given by the real part of the self energy. The

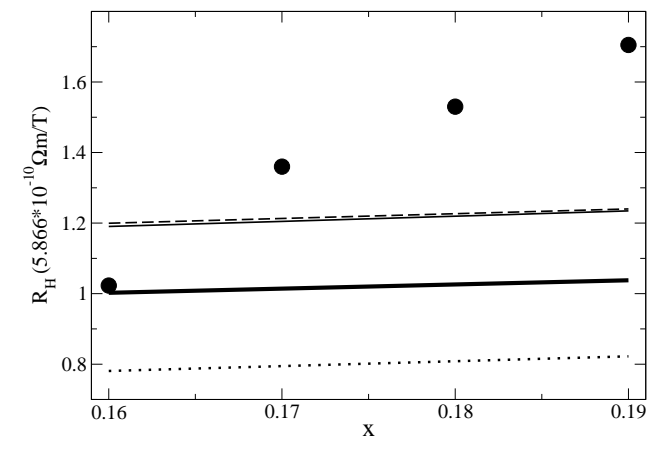

FIG. 7: Numerical results of Hall coefficient in normal state for different choices of band parameters. Heavy solid line: $t_{2}=0.32 t_{1}$ and $t_{3}=0.075 \mathrm{eV}$; dashed line: $t_{2}=0.2 t_{1}$ and $t_{3}=0.5 t_{2} ;$ dotted line: $t_{2}=0.45 t_{1}$ and $t_{3}=0.5 t_{2} ;$ light solid line: curve $1 /(1-x)$; solid circles: experimental data. ${ }^{11}$

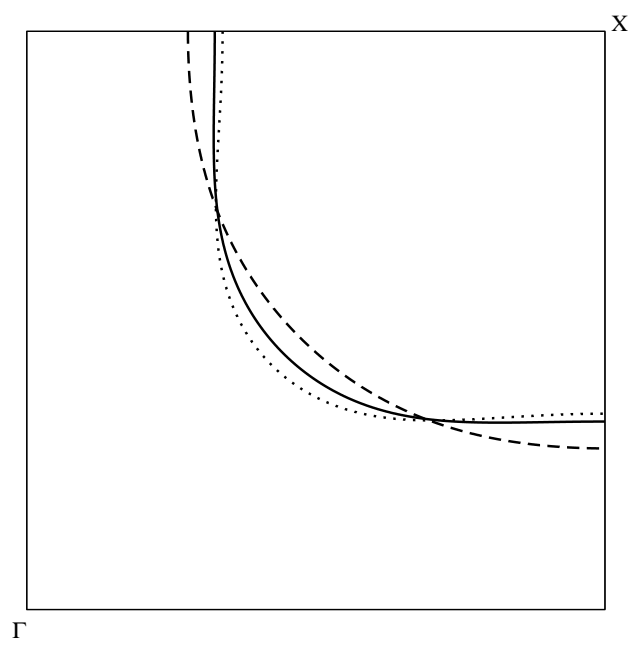

FIG. 8: Normal state Fermi surfaces in the first quadrant of Brillouin zone for different choices of band parameters at $x=0.16$. Solid line: $t_{2}=0.32 t_{1}$ and $t_{3}=0.075 \mathrm{eV}$; dashed line: $t_{2}=0.2 t_{1}$ and $t_{3}=0.5 t_{2} ;$ dotted line: $t_{2}=0.45 t_{1}$ and $t_{3}=0.5 t_{2}$.

leading approximation to this is:

$\Sigma(\mathbf{k}, i \omega)=-g^{2} T \int(d \mathbf{q}) \sum_{i \Omega_{n}} \mathcal{G}\left(\mathbf{k}+\mathbf{q}, i \omega+i \Omega_{n}\right) \mathcal{D}\left(\mathbf{q}, i \Omega_{n}\right)$

where $\mathcal{G}$ and $\mathcal{D}$ are electron and spin fluctuation Matsubara Green's functions, respectively. In our calculation, we shall take: $\mathcal{G}(\mathbf{p}, i \omega)=\left(i \omega-\varepsilon_{p}\right)^{-1}$ and $\mathcal{D}\left(\mathbf{q}, i \Omega_{n}\right)=$ $-\left(\Gamma_{q}+\left|\Omega_{n}\right|\right)^{-1}$, with $\Gamma_{q}=\Gamma\left(r+\xi_{0}^{2}(\mathbf{q}-\mathbf{Q})^{2}\right)$, where $\Gamma$ is the energy scale characteristic of spin fluctuations, $\xi_{0}$ is coherent length, $r$ characterizes the distance to criticality, and $\mathbf{Q}=(\pi, \pi)$.

This self energy for $i \omega=0$ can be integrated immediately, at $T \rightarrow 0$, if both $\varepsilon_{k+Q} / E_{0}\left(E_{0}\right.$ is an energy cutoff $)$ and $r$ are small. The result may be represented as

$$
\Sigma(\mathbf{k}, i \omega=0)=-0.5 \lambda \varepsilon_{k+Q} \ln \frac{1}{\left(r^{2}+\left(\varepsilon_{k+Q} / E_{0}\right)^{4}\right)}
$$


where, $\lambda$ is a dimensionless constant.

We can take $\varepsilon_{p}+\Sigma(\mathbf{p}, i \omega=0)$ as our new electronic energy dispersion relation, and integrate Eqs 1416 numerically. The Hall coefficient $R_{H}$ as function of $r$, for $\lambda=0.2$ (solid line) and $\lambda=0.4$ (dashed line), is shown in Fig [9] where the parameters are taken as: $t_{1}=0.38 \mathrm{eV}$, $t_{2}=0.5 t_{1}, t_{3}=0.075 \mathrm{eV}$, and $E_{0}=0.88 \mathrm{eV}$. Because the relationship between $r$ and doping has not yet established, in our numerical calculation, we keep the chemical potential fixed at the unrenormalized value for $x=0.16$. Fig[10] shows Fermi surfaces for the case $\lambda=0.2, r=0.01$ and the case $\lambda=0.4, r=0.1$, as well as the original unrenormalized one. Both of these Fermi surfaces give $R_{H}$ about $25 \%$ less than the unrenormalized value.

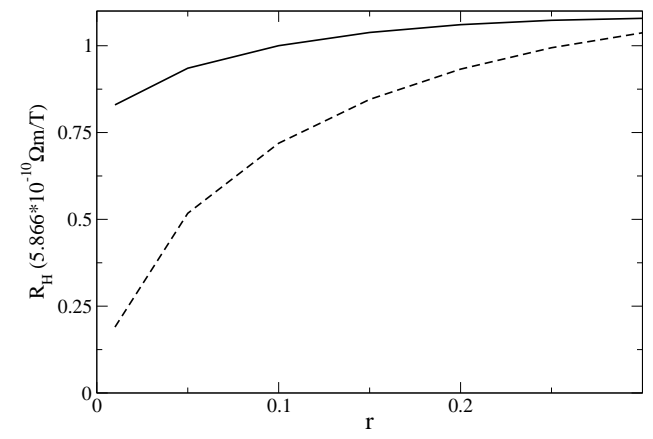

FIG. 9: Numerical result for Hall coefficient as a function of $r$ when electronic energy dispersion is $\varepsilon_{p}+\Sigma(\mathbf{p}, i \omega=0)$, for coupling constant $\lambda=0.2$ (solid line) and $\lambda=0.4$ (dashed line). Other parameters are: $t_{1}=0.38 \mathrm{eV}, t_{2}=0.5 t_{1}, t_{3}=$ $0.075 \mathrm{eV}$, the energy cutoff $E_{0}=0.88 \mathrm{eV}$.

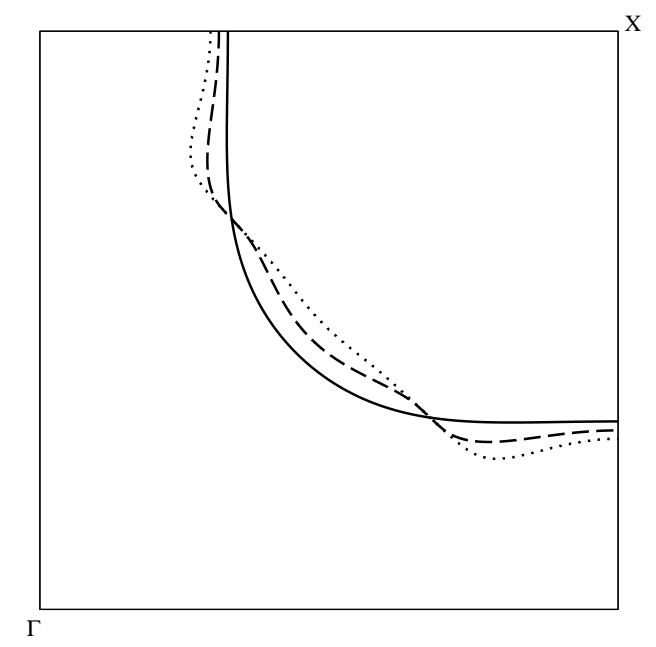

FIG. 10: Fermi surfaces in normal state, with renormalized energy dispersion relation, $E q$ [35]: $\lambda=0$ (solid line); $\lambda=0.2$ and $r=0.01$ (dashed line); $\lambda=0.4$ and $r=0.1$ (dotted line). The numerical $R_{H}$ corresponding to the dashed line is about $20 \%$ less than that corresponding to the solid line, and $R_{H}$ corresponding to dotted line is about $30 \%$ less than that of the solid line.

\section{CONCLUSION: DISCUSSION OF EXPERIMENT}

We have used mean field theory to calculate the changes in the Hall resistivity of a two dimensional system which undergoes a spin density wave instability. The model studied was chosen to be appropriate to the electron-doped cuprates. The important physics is that as $x$ is decreased below a critical value $x_{c}$, the Fermi surface is rearranged (Fig 11), leading to changes in $R_{H}$. These changes were discussed, in the context of a theory of $\mathrm{Cr}$, by Bazaliy et $a l, \frac{16}{16}$ and the related discussions, in the context of the d-density wave model of cuprates, were given by Ref. 9. The new results presented here include consideration of a wide range of dopings and gap values (Fig 3), a systematic treatment of all relevant effects (Sec. III), a discussion of the weak field-strong field crossover (Sec. IV), a treatment of precursor effects (Sec. VII), and detailed application of the model to the electron-doped cuprates (Fig 3 and conclusion). We note, unfortunately, that the specifics of the critical behavior and weak to strong field crossover will be visible (at presently accessible fields $B \lesssim 50 \mathrm{~T}$ ) only at samples doped very close $(\delta x \sim 0.005)$ of the critical doping, and at the present level of sample purity, these effects will be complicated by magnetic breakdown. The data of Ref. 11 suggest $x_{c} \approx 0.165$ while the data of Ref. 12 indicate $x_{1}>0.1$ and is most probably $\sim 0.12$. Thus the gap apparently opens quite fast with doping. However, important discrepancies remain between data and calculation. At $x<x_{1}$ our calculation shows $R_{H} \approx 1 / x$ (as expected for a Fermi surface with one electron pocket containing $x$ electrons). The data of Ref. 12 are qualitatively consistent with this trend, but are offset, suggesting that the actual doping is slightly lower than the nominal one. The $x=0.11$ data point of Ref. 11 is qualitatively inconsistent with theory. Also, the magnitude reported ${ }^{11}$ of $R_{H}$ at $x>x_{c}$ is inconsistent with theory. One expects that as $x$ is increased beyond $x_{c}, R_{H}$ should revert to the band value, $R_{H} \sim 1 /(1-x)$. While the sign and order of magnitude are correct, the numerical value is too large; there is no reasonable choice of band parameters which reproduces the magnitude to better than a factor of two.

Our work suggests several important directions for future research. On the experimental side, we suggest that our finding that $R_{H} \approx 1 / x$ for $x<x_{1}$ can be used to determine the actual doping of lightly doped samples $(x \lesssim 0.1)$. We also note that experiments which pinned down the behavior in the $x_{c}>x>x_{1}$ regime and more precisely located $x_{1}$ would be very helpful. Finally, clarifying the behavior at $x>x_{c}$ is potentially of considerable interest, as it gives (in principle) evidence of the modification of the Fermi suface by finite range SDW correlations. On the theoretical side, important directions include combining the present considerations with those of Ref. 10 to obtain a theory of the Nernst effect, and improving the theoretical treatment of the SDW precursor effects. Work on the AC Hall effect is in progress. 
Acknowledgements We thank R. Greene, Y. Dagan and M. R. Norman for helpful discussions and A. J. Schofield for drawing our attention to the strong-field crossover and the possibility of magnetic breakdown. This work was supported by NSF-DMR-0431350.
1 S. Sachdev, Rev. Mod. Phys. 75, 913 (2003).

2 P. Monthoux, A. V. Balatsky, and D. Pines, Phys. Rev. Lett. 67, 3448 (1991).

3 S. A. Kivelson, I. P. Bindloss, E. Fradkin, V. Oganesyan, J. M. Tranquada, A. Kapitulnik, and C. Howald, Rev. Mod. Phys. 75, 1201 (2003).

${ }^{4}$ C. M. Varma, Phys. Rev. Lett. 83, 3538 (1999).

${ }^{5}$ G. M. Luke et. al., Phys. Rev. B 42, 7981 (1990).

6 M. Matsuda, Y. Endoh, K. Yamada, H. Kojima, I. Tanaka, R. J. Birgeneau, M. A. Kastner, and G. Shirane, Phys. Rev. B 45, 12548 (1992).

7 P. K. Mang, O. P. Vajk, A. Arvanitaki, J. W. Lynn, and M. Greven, Phys. Rev. Lett. 93, 027002 (2004).

8 M. R. Norman, Q. Si, Ya. B. Bazaliy, and R. Ramazashvili, Phys. Rev. Lett. 90, 116601 (2003).

9 S. Chakravarty, C. Nayak, S. Tewari, and X. Yang, Phys.
Rev. Lett. 89, 277003 (2002).

10 V. Oganesyan and I. Ussishkin, Phys. Rev. B 70, 054503 (2004).

11 Y. Dagan, M. M. Qazilbash, C. P. Hill, V. N. Kulkarni, and R. L. Greene, Phys. Rev. Lett. 92, 167001 (2004).

12 Y. Onose, Y. Taguchi, K. Ishizaka, and Y. Tokura, Phys. Rev. Lett. 87, 217001/1-4 (2001).

13 R. G. Chambers, Proc. Roy. Soc. (London) A238, 344 (1957).

14 E. I. Blount, Phys. Rev. 126, 1636 (1962).

15 N. P. Ong, Phys. Rev. B 43, 193 (1991).

16 Ya. B. Bazaliy, R. Ramazashvili, Q. Si, and M. R. Norman, Phys. Rev. B 69, 144423 (2004).

17 J. Fenton and A. J. Schofield, cond-mat/0507245 